\title{
Pulmonale Spätmanifestation nach Ovarial- und Mammakarzinomen - welchen Beitrag liefert die Immunhistochemie?
}

\author{
P. Haidl ${ }^{1}$ \\ F. W. Riffelmann ${ }^{1}$ \\ P. Kemper ${ }^{1}$ \\ D. Köhler ${ }^{1}$ \\ K. M. Müller ${ }^{2}$
}

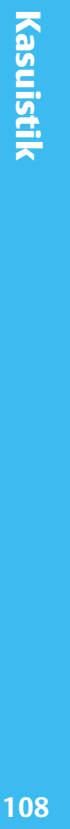

\section{Zusammenfassung}

Dargestellt werden die Krankheitsverläufe von zwei Patientinnen mit unklaren, tumorverdächtigen Lungenveränderungen. Beiden Patientinnen war gemeinsam, dass sie sowohl über 20 Packungsjahre geraucht hatten als auch vor 10 bzw. 15 Jahren an einem gynäkologischen Tumor operiert worden waren. Herkömmliche Färbeverfahren des Biopsiegutes können insbesondere bei einem Adenokarzinom nur bedingt zwischen einem primären Bronchialkarzinom oder einer Lungenmetastase eines gynäkologischen Tumors unterscheiden. Immunhistochemische Untersuchungen ermöglichten bei diesen Patientinnen die klare Diagnose später Lungenmetastasen eines Mamma- bzw. Ovarialkarzinoms nach Latenzphasen von 10 bzw. 15 Jahren. Von großer Bedeutung ist die enge Kooperation zwischen Kliniker und Pathologen, dem alle wichtigen Vorinformationen zum gezielten Einsatz seiner Verfahren zur Verfügung stehen müssen.

\section{Abstract}

The two case studies are describing 2 females with tumor-suspicious lung opacities including pleural effusions. Both patients have had a smoking history of more than 20 packyears and both underwent surgery for a breast respectively an ovarian cancer 10/15 years before. Conventional staining of the biopsies does not allow in most cases the differentiation between a primary lung carcinoma or the metastasis of an extrapulmonary tumor. Immunohistochemical techniques can give clues as to the origin of the primary tumor. The contribution of these methods in finding the correct diagnosis in the two presented cases is discussed. The cooperation between clinician and pathologist should be emphasized. The transfer of all important information is essential.

\section{Einleitung}

Im klinischen Alltag gibt es wiederholt diagnostische Probleme bei der Abgrenzung primär pulmonaler Tumoren von Metastasen, insbesondere bei Adenokarzinomen. Wir berichten über 2 Patientinnen, bei denen tumorsuspekte Lungenbefunde unter Berücksichtigung der Raucheranamnese von über 20 Packungsjahren die Diagnose einer primär pulmonalen Neoplasie nahe legten. Gleichzeitig war bei der ersten Patientin vor 15 Jahren ein Mammakarzinom und bei der zweiten Patientin vor 10 Jah- ren ein Ovarialkarzinom operativ entfernt worden. Die Diagnose von Adenokarzinomen im Lungengewebe allein basierend auf Befunden mit herkömmlichen Färbeverfahren reichen für die Differentialdiagnose zwischen einem primären pulmonalen Zweittumor und Rezidiven der bekannten gynäkologischen Tumoren nicht aus.

Anhand dieser Kasuistiken werden Bedeutung und Wert der in Kenntnis der Vorgeschichte gezielt eingesetzten immunhistochemischen Verfahren diskutiert. 


\section{Anamnese}

1986 wurde die damals 43jährige Patientin R. G. wegen eines Mammakarzinoms links brusterhaltend operiert und anschließend bestrahlt. Im Mai 2000 erfolgte eine stationäre Behandlung zur Abklärung einer arteriellen Hypertonie. Dabei wurde eine beidseitige Nebennierenhyperplasie von je ca. $2 \mathrm{~cm}$ Größe ohne endokrine Aktivität entdeckt. Gallensteine wurden nicht beschrieben. Im darauffolgenden Monat wurde Frau G. wegen einer gangränösen Cholezystitis bei Cholezystolithiasis laparotomiert. Bei Z.n. Hysterektomie wurde eine gynäkologische Untersuchung durchgeführt. Hierbei fiel ein Adnextumor links auf, gleichzeitig waren bei normalem CEA die Tumormarker CA 15-3 gering und CA 125 deutlich erhöht. Die beidseitige Mammographie ergab einen unauffälligen Befund.

Im August 2000 wurde ein $6 \mathrm{~cm}$ großes Thekofibrom des linken Ovars operativ entfernt.

Im Mai 2001 stellte sich Frau G. (Einzelhandelskauffrau) in unserer Klinik zur Abklärung einer zunehmenden Belastungsdyspnoe vor. Sie hatte vor einem Jahr den Zigarettenkonsum mit zuvor ca. 36 Packungsjahren eingestellt.

\section{Untersuchungsergebnisse}

Labor: CA 125 420kU/l (Norm < 31)

Rö-Thorax: Interstitielle Zeichnungsvermehrung der Unterfelder, Pleuraergüsse bds., keine Rundherde

CT-Thorax: Pleuraergüsse bds, mehrere Rundherde linke Lunge bis $1 \mathrm{~cm}$ Größe.

Bronchoskopie: Chronische Bronchitis, Verschluss von S6 links ohne eindeutigen exophytischen Tumor

Pleurapunktat: Exsudat, LDH $\uparrow$

\section{Oberbauchsonographie: Keine fokalen Leberläsionen}

Echokardiographie: konzentrische linksventrikuläre Hypertrophie mit diastolischer Füllungsstörung

\section{Knochenszintigraphie: Kein Hinweis auf Skelettmetastasen}

Lungenfunktion: Mittelschwere Restriktion und geringe zentrale Obstruktion, keine Einschränkung der CO-Diffusionskapazität.

Ergebnisse der Zytologie des Pleurapunktates und der Histologie der Bronchial-Biopsie: Im Pleuraerguss fanden sich zweifelsfrei Zellen eines bösartigen epitheloiden Tumors, eine Pleurakarzinose eines Adenokarzinoms ist zu favorisieren.

In der Histologie zeigten sich ebenfalls Strukturen eines Adenokarzinoms, das sich vorwiegend in den submukösen Anteilen entwickelt. Die immunhistochemischen Zusatzuntersuchungen zeigten eine massive Expression von Östrogenrezeptoren (Abb.1), die „Gegenprobe“ mit dem thyrevidalen Transkriptionsfaktor 1 (TTF-1) Antikörper verlief negativ.

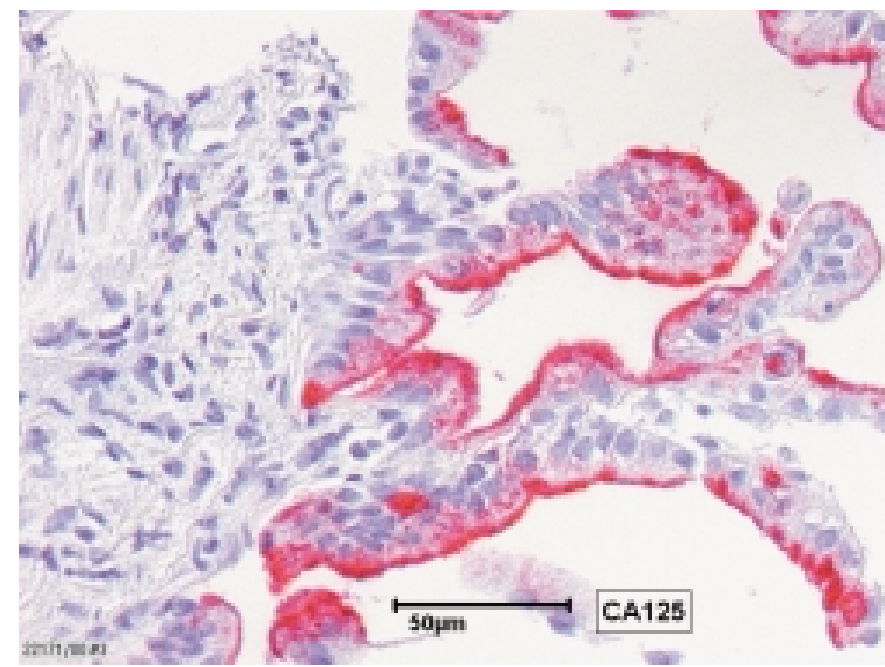

Abb. 1 Mikrofotogramm mit immunhistochemischer Darstellung von Östrogenrezeptoren in den Zellkernen der Tumorzellen als entscheidender Befund zur Einordnung des Befundes als Lungenmetastase des bekannten Mammakarzinoms.

\section{Diagnose}

Rezidiv eines Mammakarzinoms (ED 1986) mit Pleurakarzinose bds. und intrapulmonaler Metastase S6 li.

\section{Therapie und Verlauf}

Nach der Diagnosestellung wurde die Patientin in onkologische Weiterbetreuung am Heimatort entlassen. Einleitung einer Chemotherapie mit Cyclophosphamid und Epirubicin. Nach 6 Monaten partielle Remission.

\section{Kasuistik 2}

\section{Anamnese}

Im Jahre 1990 erfolgte bei der damals 58-jährigen Patientin A. R. eine Hysterektomie mit Adnexektomie wegen eines Ovarialkarzinoms mit anschließender Chemotherapie und Radiatio. Im Rahmen der onkologischen Nachbetreuung - zuletzt vor etwa 2 Jahren - wurde soweit bekannt jeweils ein unauffälliger Befund erhoben.

Im Oktober 2000 berichtete die Patientin bei der Aufnahme, dass etwa seit $1 / 2$ Jahr häufiger Husten auftritt, der sich nach einem Urlaub an der Nordsee Anfang Oktober deutlich verschlechtert habe; nur wenig weißlicher Auswurf. Darüber hinaus trat in den letzten Wochen eine zunehmende Belastungdyspnoe auf, zuletzt sei nur noch 1 Etage Treppensteigen möglich gewesen. Keine nächtliche Dyspnoe. Beinödeme oder Schmerzen bestanden nicht.

Exraucherin seit 10 Jahren, zuvor sind mindestens 20-30 Packungsjahre anzunehmen.

\section{Aufnahmeuntersuchung}

68-jährige Patientin, $159 \mathrm{~cm}, 60 \mathrm{~kg}$, in deutlich reduziertem $\mathrm{AZ}$, Dyspnoe beim An-und Auskleiden, knisternde RG über den mittleren und basalen Lungenabschnitten, Leber $3 \mathrm{QF}$ unter dem Rippenbogen tastbar. 


\section{Untersuchungsergebnisse}

Labor: pathologisch: Leukozyten 13700 und 8700, CRP 7,94 und $3,34 \mathrm{mg} \%$ (Norm $<0,6 \mathrm{mg} \%$ )

Rö-Thorax: Ausgedehnte alveoläre Infiltrationsmuster beidseits im Bereich von Oberlappen, Mittellappen und Lingula, z.T. positives Bronchopneumogramm, keine Stauungszeichen

CT-Thorax: In beiden Lungenhälften ausgedehnte zystische Läsionen mit z.T. sehr kräftigen Wandungen, große kavernös imponierende Läsion im rechten Unterlappen, zusätzlich Infiltrate mit positiven Bronchopneumogramm (Abb. 2)

Abdomensonographie: Hepatomegalie, keine fokalen Läsionen

Lungenfunktion: Geringe Restriktion (inVK 1,7l, 68\% Soll, TLC 80\% Soll)

BGA: Partialinsuffizienz bei Hyperventilation $\mathrm{pO}_{2} 63 \mathrm{~mm} \mathrm{Hg}$, $\mathrm{pCO}_{2} 27 \mathrm{~mm} \mathrm{Hg}$

Bronchoskopie: Mäßig ausgeprägte chronische Bronchitis, kein endobronchial sichtbarer Tumor, BAL in der Lingula, transbronchiale Biopsie aus der Lingula und S3 li.

BAL: Massive Granulozytose 70\% bei normaler Gesamtzellzahl, regressiv veränderte, teilweise zusammengelagerte Zellformen, die tumorverdächtig sind.

Bakteriologie: Keine pathogenen Keime einschließlich säurefester Stäbchen.

Transbronchiale Biopsie: Zunächst Bild eines bronchoalveolären Karzinoms. Die immunhistochemischen Untersuchungen zeigten keine Reaktion auf TTF-1 sowie Surfactant-Protein A und B. Dagegen positive Reaktion mit einem Antikörper gegen CA 125 (Abb. 3).

\section{Diagnose}

Diffuse pulmonale Metastasierung eines Ovarialkarzinoms (ED 1990)

\section{Verlauf}

Wegen des schlechten Allgemeinzustandes erfolgte bei Vorliegen infiltrativer Veränderungen zunächst eine breite antimikrobielle Therapie mit Clarithromycin, Clindamycin und Ceftazidim sowie Itraconazol. Nach Diagnosestellung erfolgte die Weiterbehandlung in einem onkologischen Zentrum mit einer palliativen Chemotherapie mit insgesamt 2 Zyklen Cisplatin/Cyclophosphamid. Zwischen den Zyklen wurde eine erneute Breitbandantibiose wegen neuer pneumonischer Infiltrate erforderlich. Infolge einer progredienten Verschlechterung der Grundkrankheit verstarb die Patientin am 15.1.01.

\section{Diskussion}

Bei den mit klinischen Untersuchungsverfahren nur unzureichend zu klärenden Lungenerkrankungen erhofft man sich vom Pathologen eine möglichst klare Aussage zu Art, Aktualität und Dignität morphologisch fassbarer Strukturanomalien.

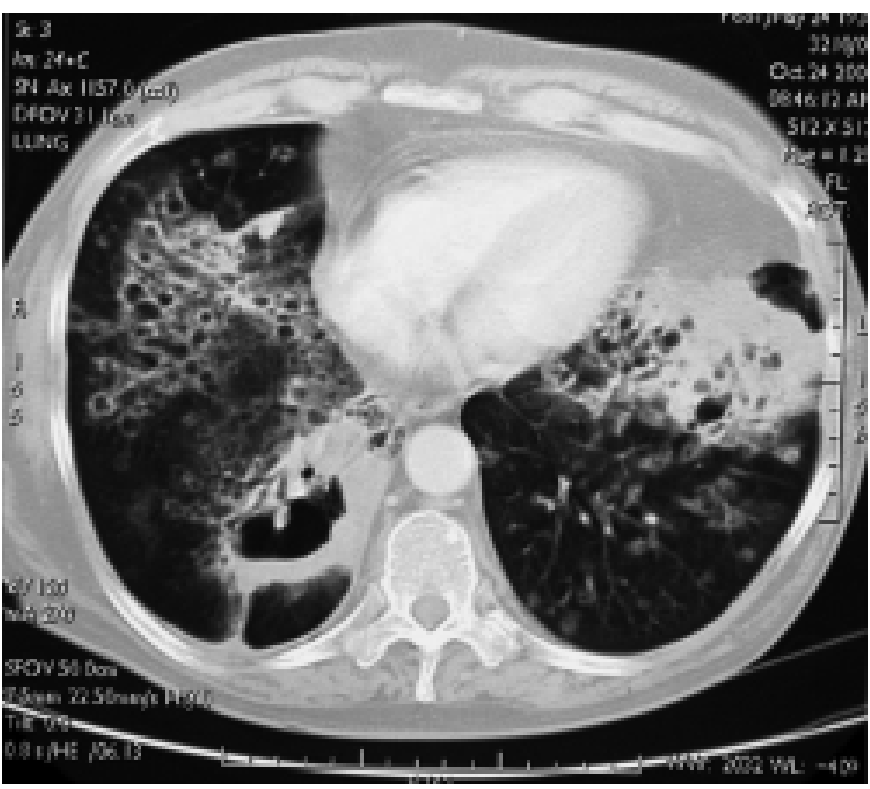

Abb. 2 CT-Thorax-Schicht: bds. ausgedehnte zystische Läsionen mit zum Teil kräftigen Wandungen, kavernöse Veränderung im re. Unterlappen, infiltrative Veränderungen re. Unterlappen.

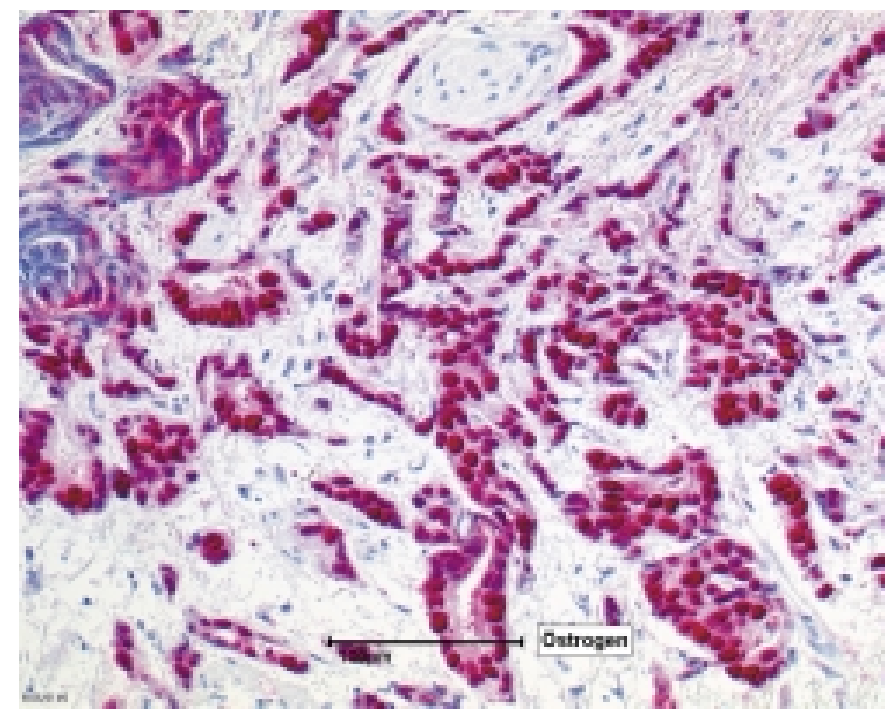

Abb. 3 Mikrofotogramm mit immunhistochemischer Erfassung von CA 125 bevorzugt in den Randabschnitten der im Bereich der Alveolarwand wachsenden Tumorzellen - als Hinweis auf die Lungenmetastasierung eines bekannten Ovarialkarzinoms.

Bei den häufigen bösartigen Lungentumoren ist zur Zeit für die Klinik die Einordnung bei einem vorwiegend kleinzelligen oder nicht-kleinzelligen Tumor für die Therapie entscheidend. Dabei darf nicht übersehen werden, dass grundsätzlich jeder Tumor Zellen sehr unterschiedlicher Größe und Differenzierungsvarianten als Ausdruck einer großen - heute auch molekularbiologisch fassbaren - Heterogenität aufweist. In 50\% der phänotypischen nicht-kleinzelligen Lungentumoren sind Differenzierungvarianten in quantitativ und qualitativ unterschiedlicher Form vorhanden [1]. Diese Beobachtung kann u. a. die unvorhersehbaren Unterschiede im Ansprechen auf eine Chemotherapie erklären.

Anders stellt sich die Frage bei den vergleichsweise selteneren metastatischen Lungenprozessen. Bei der Aufarbeitung von 
Biopsie- und Operationsgut mit herkömmlichen Färbeverfahren kann man nur bedingt den Verdacht auf Lungenmetastasen bei „abnormen“ Wachstumsmustern ableiten. So kann die Beurteilung der Vaskularisierung des Tumors ein Diagnosebaustein sein [2]. Primäre Lungentumoren werden über die Äste des nutritiven Kreislaufs, d. h. die Bronchialarterien, versorgt, die teils direkt hilifugal in das Tumorzentrum einwachsen und zahlreiche Anastomosen ausbilden [3]. Außerdem kommt es zur Kompression der Äste des funktionellen Kreislaufs, so dass schon T1-Tumoren ausgedehnte pulmonalarterielle Perfusionsstörungen mit Ausschaltung ganzer Lungensegmente bewirken. In der Lungenperfusionsszintigraphie kommt dies dadurch zum Ausdruck, dass der Perfusionsausfall im Tumorbett größer als der eigentliche Tumorkennschatten ist.

Für einige Tumorformen gibt es aber in der heute verfügbaren breiten Palette immunhistochemisch fassbarer Antigenprofile die Möglichkeit zur nahezu sicheren Einordnung der Tumorbefunde in den Lungen als Metastasen (Tab. 1) [4].

Tab. 1 Antikörper für die Differenzierung primärer Lungentumoren versus Lungenmetastasen extrathorakaler Tumoren

\begin{tabular}{ll}
\hline Primärtumor & Antigen \\
\hline $\begin{array}{l}\text { Mammakarzinom } \\
\text { Prostatakarzinom }\end{array}$ & Östrogen-/Progesteronrezeptoren \\
\hline malignes Melanom & prostataspezifisches Antigen \\
\hline $\begin{array}{l}\text { Ovarialkarzinom } \\
\text { Schilddrüsenkarzinome } \\
\text { (papillär, follikulär) }\end{array}$ & CA 125 \\
\hline $\begin{array}{l}\text { Hodentumor } \\
\text { (Seminom, Dysgerminom) }\end{array}$ & Thyreoglobulin \\
\hline Nierenzellkarzinom & plazentare alkalische Phosphatase (PLAP) \\
\hline Lymphom & proximal nephrogenic Antigen (PNRA) \\
& Leucocyte common Antigen (LCA) \\
& CD 3 T-Zell Marker \\
\hline
\end{tabular}

Die Wahrscheinlichkeit von Metastasen kann durch negative immunhistochemische Befunde für charakteristische Expressionsprofile bei primären Lungentumoren (Tab. 2) zusätzlich erhärtet werden. Heute stehen dem Pathologen mehrere 100 Antikörper zum Nachweis der Expression zellulärer und nukleärer Proteine zur Verfügung. Aber nur bei vergleichsweise wenigen Lungenmetastasen führen die gezielt eingesetzten immunhistochemischen Zusatzuntersuchungen zu eindeutigen Befunden im Sinne eines spezifischen Tumormarkers. Bei der Ermittlung von Sensitivität und Spezifität müssen mögliche technische Fehler bei den in zahlreichen Schritten ablaufenden immunhistochemischen Reaktionen Berücksichtigung finden. Gelegentlich ergeben sich auch durch die Bestimmung der Ko-Expression verschiedener Antigene wertvolle Zusatzinformationen mit Hinweisen auf den Primärtumor. Von entscheidender Bedeutung aus zeitlichen und aus Kostengründen ist es daher, dass dem Pathologen bereits für die Primärdiagnose von Biopsiegut ausreichende Informationen zur Verfügung gestellt werden. Dies gilt selbstverständlich auch für bekannte primär extrapulmonale Tumorleiden in Zeitintervallen von mehr als 5 und 10 Jahren vor Abklärung des Lungenbefundes.
Tab. 2 Antikörperpanel für die Diagnostik primärer Lungentumoren

\begin{tabular}{|c|c|}
\hline histomorphologischer Tumortyp & Antikörper \\
\hline Adenokarzinom & $\begin{array}{l}\text { TTF } 1 \text { thyreoidaler Transkriptionsfaktor } \\
\text { SP-B Surfactantprotein, Pro SP-B Vor- } \\
\text { stufe }\end{array}$ \\
\hline Plattenepithelkarzinom & kein spezifischer Marker \\
\hline \multicolumn{2}{|l|}{ neuroendokrine Tumoren } \\
\hline kleinzelliges Karzinom & CD 56 LCA negativ (DD Lymphom) \\
\hline typische und atypische Karzinoide & CD 56 Synaptophysin, Chromogranin \\
\hline $\begin{array}{l}\text { großzelliges neuroendokrines } \\
\text { Karzinom }\end{array}$ & CD 56 \\
\hline
\end{tabular}

Im ersten von uns beschriebenen Krankheitsfall war die Entfernung eines Mammakarzinoms vor 15 Jahren bei der seinerzeit erst 44 Jahre alten Patientin erfolgt. Nach den anamnestischen Befunden eines hohen Zigarettenkonsums kam als Ursache des Pleuraergusses bei gleichzeitig suspektem endobronchialen Befund auch ein Bronchialkarzinom infrage. Die immunhistochemischen Befunde der in nahezu allen Zellen nachweisbaren Östrogen- und Progesteron-Rezeptoren dürfen als Beweis für einen vergleichsweise späten metastatischen Prozess in der Lunge des vor 15 Jahren gesicherten Mammakarzinoms gelten.

Der zweite Fall zeigt das histologische Wachstumsmuster eines hoch differenzierten, teils papillär wachsenden Adenokarzinoms. Diesen Tumortyp gibt es durchaus als Variante primärer Adenokarzinome der Lunge und die erste Diagnose lautete auch: V.a. bronchoalveoläres Karzinom. Die Einordnung dieses pulmonalen Tumors als Metastase ergibt sich aus den Ergebnissen mit positiven als auch negativen immunhistochemischen Befunden. Im Wissen um das vor 10 Jahren operierte Ovarialkarzinom ließ sich ein überzeugend CA 125-positiver, membranös apikaler Befund in $80 \%$ der Tumorzellen belegen. Durch gleichzeitig negative Befunde bei der Prüfung der Expression von Surfactant-Proteinen $\mathrm{A}$ und $\mathrm{B}$ sowie des in pulmonalen Adenokarzinomen häufig positiven thyreoidalen Transkriptionsfaktors 1 (TTF 1) [5] konnte die Lungenmetastase eines Ovarialkarzinoms, in Abgrenzung zu einem primären pulmonalen Adenokarzinom, als sehr wahrscheinlich abgeleitet werden.

In beiden Fällen waren Informationen zu den früheren Tumoren von besonderer Bedeutung, damit bei der breiten Palette möglicher Zusatzanalysen zur Tumorcharakterisierung als Metastasen die infrage kommenden Antikörper gezielt und damit zeit- und kostensparend eingesetzt werden konnten. Die Ergebnisse waren dann von wegweisender Bedeutung in Bezug auf Therapie und Prognose.

Beide Krankheitsbilder zeigen, dass bei Fragestellungen sekundärer pulmonaler und pleuraler Tumoren eine enge Abstimmung zwischen Klinik und Pathologie notwendig ist. 
${ }^{1}$ Müller KM. Neues zur Pathologie der Lungentumoren. Verh Dtsch Ges Path 1999; 83: $168-183$

${ }^{2}$ Reitemeyer E, Bordt J, Müller KM. Angiographische Befunde bei Lungenmetastasen. Verh Dtsch Ges Path 1984; 68: 224-229

3 Borek Z, Polak J, Macholda F. Die nutritive Vaskularisation des Bronchialkarzinoms im arteriographischen Bild. Radiologie 1970; 10: $317-329$
${ }^{4}$ Fisseler-Eckhoff A, Müller KM. Differenzialdiagnose primärer Lungentumoren und pulmonaler Metastasen. Verh Dtsch Ges Path 2000; 84: $106-117$

${ }^{5}$ Kaufmann O, Dietel M. Pathohistologische Diagnostik bei Karzinommetastasen eines unbekannten Primärtumors. Onkologie 1997; 3 : $342-349$

\section{Buchbesprechung}

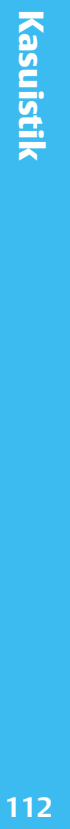

\section{Die Lungenfunktion - Methodik und klinische Anwendung 6. völlig neu gestaltete Ausgabe}

Ulmer, W. T., D. Nolte, J. Lecheler, T. Schäfer (Hrsg.)

XIV, 178 S., 85 Abb., 23 Tab.; Geb.

Georg Thieme Verlag Stuttgart; 2001

ISBN 3-13-448 806-X

Preis: DM 199,00/EUR 101,75/ATS 1.453,00/CHF 177,00

Erst seit den dreißiger Jahren des vergangenen Jh. haben Physiologie und Pathophysiologie der Atmung für den Kliniker, die Intensivmedizin und die Anästhesiologie wie für die operationelle Medizin im Überdruck, für die submarine und die Luft- und Raumfahrtmedizin zunehmend an Bedeutung gewonnen. Die laufend verbesserten funktionsdiagnostischen Verfahren sind heute unverzichtbar.

Neben den - heute wohl nur den Älteren noch bekannten - Standardwerken (z. B. Rossier und Bühlmann (1956)); Comroe, Forster, DuBois, Briscoe und Carlsen (1964); Comroe (1965); Burton (1969); Cherniak (1977) hat sich seit 1970 im deutschen Sprachraum „der Ulmer“ seinen festen Platz erorbert. Einer italienischen Ausgabe (1975) folgten jeweils überarbeitete und ergänzte Auflagen 1976, 1983, 1986 und 1991.

Die Vertiefung unseres Grundlagenwissens und die Verfeinerung der Diagnostik, neue (Sub-)Fachgebiete in Allergologie, Pädiatrie und Pneumologie haben die Hrsg. und ihre 8 Mitarbeiter veranlasst, die vorliegende Ausgabe völlig neu zu schreiben, die bisherige Grundstruktur jedoch beizubehalten. Didaktisch eindrucksvoll vermitteln sie, wie die Lungenfunktionsdiagnostik zwar von hochperfektionierten Geräten geleistet wird, dass aber der Untersucher selbst die Methodik voll beherrschen muss, um die digital gebotenen Messgrößen korrekt zu bewerten und klinisch zu interpretieren. Allein die subtile Kenntnis der Zusammenhänge ist Voraussetzung für Diagnose wie Behandlung von Störungen (Erkrankungen) des Funktionskomplexes Herz-Kreislauf-Atmung. Dem trägt nunmehr auch die Erweiterung um Leitlinien zu schlafassoziierten Störungen, Besonderheiten der Lungenkrankheiten im Kindesalter und der vielfältigen Asthmavariablen Rechnung.
Das Werk ist in drei Sachgebiete gegliedert, denen ein Verzeichnis der verwendeten Abkürzungen und Akronyme vorangestellt ist.

In „I. Grundlagen“ werden in 6 Kapiteln Atemmechanik (Strömungs- und Gewebswiderstand, Dehnbarkeit); Physiologie und Pathophysiologie der Atemwege; Ventilation und Atmungsregulation; Lungenkreislauf und Gasaustausch; Schlafassoziierte Störungen der Atmung und Besonderheiten im Kindesalter behandelt. Gebiet „II. Methodik der Funktionsdiagnostik“ vermittelt in 10 Kapiteln nach einer Einführung die Spirometrie und Ganzkörperplethysmographie sowie deren Korrelationen und Sollwerte; Oszilloresistometrie, Unterbrechermethode und $\mathrm{P}_{0,1}$-Messung; Messung der Lungendehnbarkeit; Diffusion (Transferfaktor TLCO), Blutgas- und Atemgasanalyse in Ruhe und unter Belastung; Inhalative Provokationstests, Bronchodilatationstests und $\mathrm{CO}_{2}$-Antwortversuche; Analyse schlafassoziierter Störungen und methodische Besonderheiten im Kindesalter. Insgesamt ein umfassender Überblick über die funktionsanalytischen Methoden für Diagnose, Therapie (und deren Kontrolle) und für die Begutachtung.

Der „III. Anhang“ bietet ausführliche Tabellen der Korrektur- und Umrechnungsfaktoren; Referenzwerte; gebräuchliche Symbole für Atmung und Kreislauf und die Vielzahl der Abkürzungen. Das Verzeichnis der weiterführenden, auch internationalen Literatur(stellen) beschränkt sich bewusst auf das Wesentliche (aus der heutigen Literaturschwemme). Es ist zu begrüßen, dass auch einige wenige der „deutschen Klassiker“ des Gebietes wie z.B. Große-Brockhoff, Hayek und Pflüger nicht unerwähnt bleiben. Die einzelnen Kapitel sind durch zweifarbige Graphiken und Tabellen illustriert; einprägsam vermitteln hervorgehobene Kernsätze, Definitionen, Formeln und Sollwerte die Wechselbeziehungen von Hämodynamik und Lungenfunktion. Ein „Sachverzeichnis“ rundet den „neuen Ulmer“, nun auch in größerem Formate und in aktueller Qualität ab. Insgesamt ein imponierendes, in sich geschlossenes Standardwerk mit einer Fülle von Einzeldaten und experimentellen Ergebnissen: ein instruktives Lehrbuch, Leitfaden für die klinische Anwendung und Nachschlagewerk zugleich. 